\title{
EL INGLÉS Y SU IMPORTANCIA EN LA INVESTIGACIÓN CIENTÍFICA: ALGUNAS REFLEXIONES
}

\section{ENGLISH AND ITS IMPORTANCE IN SCIENTIFIC RESEARCH: SOME REFLECTIONS}

\author{
NIÑO-PUELLO, MIRYAM ${ }^{1 *}$ MSc. \\ ${ }^{1}$ Universidad de Sucre, Departamento de Educación y Humanidades, Facultad de \\ Educación y Ciencias, Sincelejo, Colombia. \\ ${ }^{*}$ Correspondencia: miryampuello@yahoo.com
}

Recibido: 08-04-2013; Aceptado: 04-05-2013.

\section{Resumen}

La publicación de resultados de investigaciones científicas es actualmente el reto más grande de cualquier investigador sin importar sí su producción pertenece a las ciencias puras o humanas. Ser capaz de comunicarse con el mundo académico y sus seguidores es un requisito fundamental para sobresalir en este planeta que la ciencia y la tecnología han convertido en una pequeña aldea. En este artículo se describe la importancia del inglés como lengua internacional para la investigación científica. Indudablemente, el inglés es un idioma que está generando grandes oportunidades no solo para nuestras vidas como profesionales sino también como agentes trasmisores de una cultura mundial orientada hacia la búsqueda del bien común. El impacto de la investigación como factor transformador de nuestras sociedades tampoco pude desconocerse, y es aquí donde una lengua mundial como el inglés fortalece las posibilidades de desarrollo científico, cultural, económico y humanístico.

Palabras claves: investigación científica, resultados, inglés, ciencia dura, ciencia blanda

\section{Abstract}

Nowadays, the publication of scientific research results is the highest challenge for researchers independently of the kind of science his or her work belongs: hard or soft one. Be able to be in touch with the academic world and its followers is a fundamental requirement to stand out in a planet that science and technology have become in a small village. In this article, the importance of English as an international language for the scientific research is described. Undoubtedly, English is the language that foster great opportunities not only for our professional lives but also as transmitter agents of a worldwide culture focused in a common goal. We cannot ignore the impact of research as a factor able to improve our 
societies and it is precisely here when a universal language like English strengths the possibilities of a scientific, cultural, economic and humanistic development.

Key words: scientific research, outcomes, English, hard science, soft science.

\section{Introducción}

El inglés no es el idioma más hablado en el mundo, pero si el más importante. Estimaciones recientes de KRISS TALAAT (2010) presentadas en Wikipedia sugieren que aproximadamente 335 millones de personas hablan inglés como su primer idioma y otros 430 millones lo hablan como su segunda lengua. Por otro lado, la página web www.ukstudies.com considera que más del $80 \%$ de la información electrónica almacenada alrededor del planeta está en inglés y las dos terceras partes de los científicos de la tierra leen en dicha lengua. Otro dato interesante también planteado en dicha web es que del total estimado de 2400 millones de usuarios de internet, un $80 \%$ se comunica actualmente en inglés. Además, éste está presente en la vida cultural, social, política y económica de la mayoría de los países del orbe.

El objetivo de este artículo es identificar y analizar la importancia del inglés en la producción científica escrita. A continuación, se abordarán aspectos históricos que han convertido al inglés en la lengua franca de los investigadores, su importancia general dentro del campo de la investigación científica así como las áreas específicas de investigación que lo utilizan en sus publicaciones y su comparación con otros idiomas.

\section{Origen del idioma inglés}

Con la llegada de tres tribus Germánicas a las islas británicas en los años 500 AC, comenzó la historia del idioma inglés: Anglos, Sajones y Jutos cruzaron el Mar del Norte desde la hoy llamada Dinamarca y el norte de Alemania. Los Anglos recibieron ese nombre debido a su tierra de origen Engle o Angels. Ellos denominaron a su propia lengua Englisc, de la cual se derivó English o inglés (CRYSTAL, 1977, 2004). Antes de la llegada de los invasores, los habitantes de Gran Bretaña hablaban un idioma de origen Céltico. A medida que las tribus germánicas se expandían por el país se desarrollaron cuatro dialectos: Northumbrian, Mercian, West Saxon y Kentish (CRYSTAL, 2004). Durante los años 700 y 800 , la cultura y el dialecto de Northumbrian dominó, pero la invasión de los Vikingos en los años 900 puso fin a esa dominación, sólo el reino de Wessex (la parte sudoccidental de Gran Bretaña) permaneció como territorio independiente (CRYSTAL, 1977, 2004). 
Alrededor de la décima centuria, el dialecto sajón del Oeste se convirtió en el idioma oficial. Existen muestras del inglés antiguo escritos en su mayoría en el alfabeto Rúnico, el cual tuvo su origen en las lenguas escandinavas (CRYSTAL, 2004). El alfabeto latino fue traído desde Irlanda por los misioneros cristianos y fue adoptado desde entonces (CRYSTAL, 2004).

El vocabulario del Antiguo Inglés estaba conformado por una mezcla de palabras Anglo-Sajonas con términos prestados de las lenguas Escandinavas (danés y noruego) y del latín. Es así que el Latín introdujo al Inglés palabras como calle (street), cocina (kitchen), queso (cheese), vino (wine), ángel (angel), obispo (bishop), entre otras. Los Vikingos por su parte agregaron palabras noruegas como: cielo (sky), huevo (egg), piel (skin), ventana (window), esposo (husband), habilidad (skill), impar (odd), conseguir (get), dar (give), tomar (take), llamar (call). Las palabras celtas aún subsisten, principalmente en nombre de lugares y ríos (Devon, Dover, Kent, Trent, Severn, Avon, Thames).

Pero años más tarde, en 1066 los Normandos conquistaron Gran Bretaña y el francés se convirtió en el idioma de la aristocracia normanda y es esa la razón de palabras como: close (shut), reply (answer), anual (yearly), demand (ask), desire (wish) (CRYSTAL, 2004). Sin embargo, en el siglo XIV el inglés volvió a ser la lengua dominante en Gran Bretaña, ya que en 1399 Henry IV, cuya lengua era el inglés se convirtió en el primer rey de Inglaterra. En 1476, William Caxton introdujo la primera máquina de imprenta lo cual extendió la influencia del dialecto London Standard, el más usado en ese momento, por todo el país. Se establecieron normas de escritura y gramática llegándose a publicar en 1604 el primer diccionario del idioma inglés. A partir del siglo XVI, la expansión del imperio británico, conjuntamente con el Renacimiento, trajo consigo muchas palabras de origen foráneo que se incorporaron al inglés, directa o indirectamente. Se crearon nuevas palabras a un ritmo cada vez más intenso (CRYSTAL, 1977; KATSIAVRIADES y QURESHI, 2002; THOMPSON y BALKWILL, 2006).

El idioma inglés evolucionó con la historia del planeta y hoy podemos hablar de un idioma internacional que es definitivo en la vida mundial en todas las áreas sociales, políticas, económicas, culturales, tecnológicas y científicas. En la Fig. 1 se muestra las diversidades de inglés que podemos encontrar hoy en día.

\section{El inglés y la investigación científica}

A continuación se plantea la importancia del inglés en la investigación y en las publicaciones científicas. El uso de esta lengua internacional en el área de la 
ciencia está bien documentado. El inglés ha trascendido culturas y ha influenciado desde la moda hasta las tradiciones y estilos de vida. CRYSTAL (2004), sostiene que la importancia del inglés en las publicaciones científicas se remonta a la Revolución Industrial. Según este autor, dos tercios de los científicos y tecnólogos que hicieron posible dicho movimiento usaban el inglés (al principio británicos y más tarde americanos) como lengua materna, así que cualquiera que deseara conocer acerca de los últimos avances no tenía otra opción sino adquirir la competencia en inglés, ya sea directamente o través de la traducción.

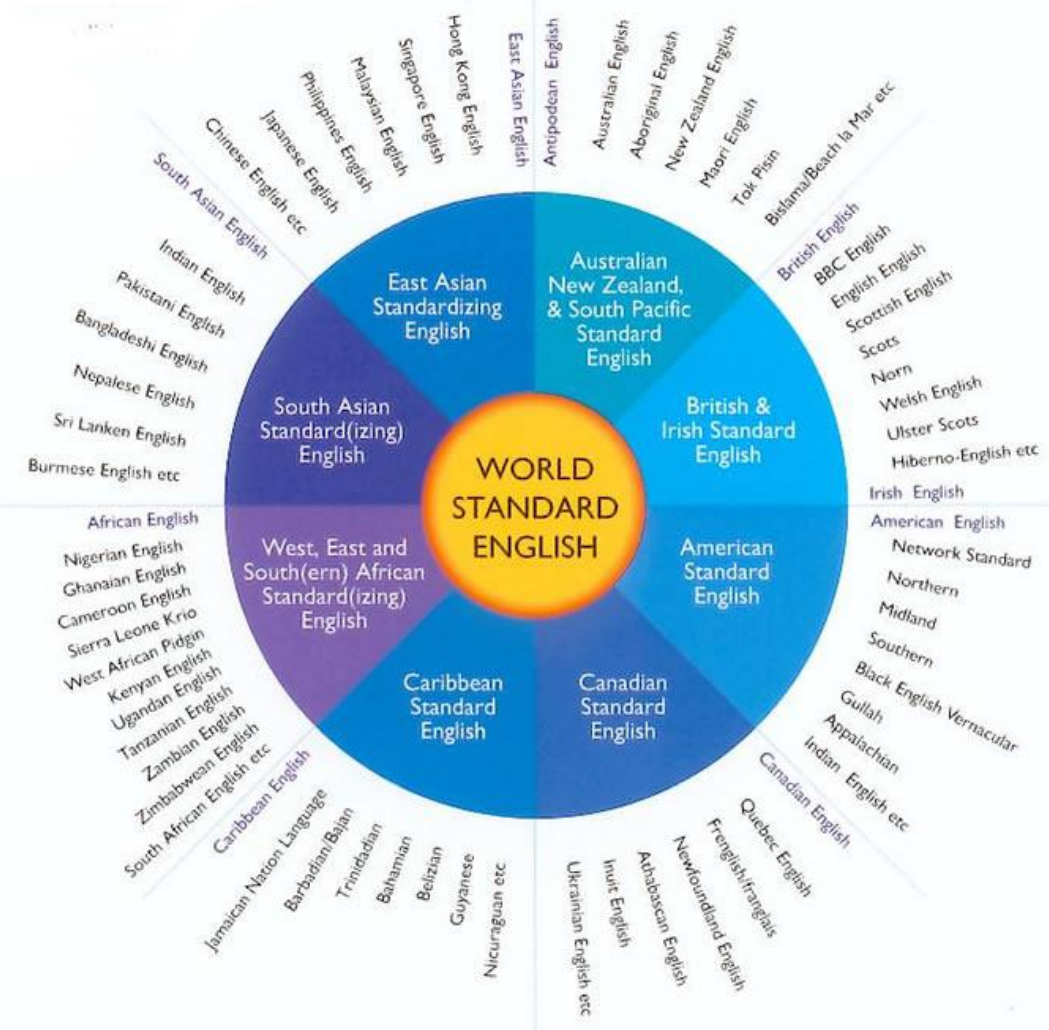

Figura 1. Diversidad del idioma inglés. Fuente: http://www.britemb.cl/100qts/100qts_idioma_56.html

Para 1953, SAVORY, citado por CRYSTAL (2004), conceptúo que el inglés ya daba señales de convertirse en el idioma de la ciencia. De otra parte, SUBEDI dijo que en 1931 VLADIMIR N. IPATIEFF, un químico ruso-americano comenzó a tomar clases de inglés a la edad de 64 años. Él era ya un reconocido científico pero tenía que aprender inglés a esa edad para continuar su investigación en los Estados Unidos. Probablemente estaba bajo la influencia del dictamen "publicar o perecer", muy común en el campo de la investigación. 
MICHAEL FARADAY (http://neltachoutari.wordpress.com/2010/05/01) dice que cualquier investigador tiene que seguir tres pasos: "trabajar, analizar y publicar". Todas tres igualmente relevantes. Sin embargo, la importancia del idioma inglés aparece en la tercera -publicar. La producción real de cualquier científico se mide por su impacto, de ahí que el nivel de las revistas especializadas se determina por el mismo factor. Se dice que es más importante el número de personas que citen nuestros trabajos que la cantidad de trabajos que escribamos. Para hacer nuestras publicaciones accesibles a un gran grupo de lectores, debemos publicar nuestros resultados en un idioma comprendido por mucha población.

De acuerdo con SUBEDI (2012), en Japón, el inglés se está convirtiendo en el idioma de las ciencias básicas lo cual ha conllevado a la gradual desaparición de publicaciones en japonés. El mismo autor asegura que RIKEN, uno de los grupos investigativos más conocidos del Japón, ha manifestado que sus científicos publicaron casi 2.000 reportes originales en inglés en 2005 y solo 174 en japonés. Otro estudio muestra que las compañías editoras en Japón cobran a los investigadores de 500 a 800 por manuscritos, un curso de idiomas de 10 semanas podría costar 2000 dolares. Estos costos son carga adicional y enlentecen las actividades científicas en los laboratorios. Igualmente, este mismo autor considera que el nivel de proficiencia en inglés en términos orales de un investigador facilitara o dificultara su participación en congresos internacionales. A pesar de sus buenos resultados investigativos, algunas veces los científicos están tan nerviosos en sus presentaciones debido a la dificultad que tienen para expresar claramente sus ideas en inglés, mientras que aquellos que han realizado sus cursos de este idioma se notan más confiados incluso cuando el mérito de sus investigaciones no sea de alto nivel. (Nelta Choutari " English and Scientific Research: Some Reflections Comments Feed).

Otro caso donde la proficiencia en inglés juega un rol importante es en la preparación de propuestas investigativas. Una prometedora investigación podría ser rechazada por la carencia de coherencia y cohesión de su contenido, si bien es cierto que un editor podría colaborar, muchas veces éste podría no conocer las ideas técnicas del proyecto. Considerando lo anterior muchos países europeos y asiáticos están gradualmente adoptando el inglés como la lengua de la ciencia (KIRCHIK et al., 2012; VAN WEIJEN, 2012).

El inglés es considerado la lengua franca de la comunidad científica. Por ejemplo $80 \%$ de las revistas especializadas indexadas en Scopus (base de datos de investigaciones científicas) son publicadas en inglés. Su adopción como lengua universal de las ciencias es debida en parte a factores económicos e histórico políticos, los cuales favorecieron al inglés por encima de lenguas potenciales 
como el alemán, chino, francés, ruso y español. TARDY (2004) establece que el alemán fue el idioma preponderante en la primera parte del siglo XX; el mismo autor dice que a pesar que actualmente el inglés es el idioma principal de la comunicación científica internacional, algunos investigadores siguen publicando en otros idiomas.

En un primer estudio realizado entre 1996 y 2007, VAN WEIJEN (2012) confirmó la preferencia de los investigadores por inglés ante sus idiomas nativos en la mayoría de los países de Europa occidental incluidos en la muestra. La cantidad de publicación desde el inglés hasta el alemán y del inglés al italiano fue particularmente alta, comparada con aquellos otros países del estudio (Alemania, Francia, España y la Federación Rusa). La Fig. 2 muestra el número de artículos publicados en revistas especializadas publicadas en inglés y en cada idioma oficial del país.

El análisis se extendió en un segundo estudio hacia países como Brasil y China por ser considerados por el autor como economías en ascenso investigativo, lo cual es confirmado con el hecho de que la tasa de crecimiento anual para los artículos indexados publicados en Scopus entre 1996 y 2011 en Brasil fueron del $13 \%$ y del $19 \%$ en China, lo cual es muy superior al 3 y $5 \%$ que es lo usualmente esperado (VAN WEIJEN, 2012).

La Fig. 2 muestra cómo, en contraste con el estudio original, el uso del inglés continuó aumentando fuertemente en Holanda, Italia y Rusia durante los pasados 4 años, también se incrementó un poco en Alemania, pero permaneció relativamente estable en Francia, España y China. Sin embargo, la diferencia entre el portugués y el inglés en Brasil está claramente decreciendo, aunque esto podría ser en parte a un incremento en el cubrimiento de las revistas especializadas publicadas en portugués en lugar de inglés en Scopus. Aun así el crecimiento del uso del inglés es notorio todo el tiempo (VAN WEIJEN, 2012).

VAN WEIJEN, (2012), intenta dar respuesta a la pregunta de sí había áreas de investigación especificas en las cuales los investigadores aún publicaban regularmente en su propios idiomas en lugar del inglés; para dar respuesta los investigadores del estudio en mención realizaron una búsqueda en Scopus para determinar el número de artículos publicados en cada uno de los idiomas seleccionados entre 1996 y 2011. Las lenguas incluidas en la búsqueda fueron las mismas presentados en la Fig. 2, con la adición del inglés, de esta manera, lograron establecer la comparación que buscaban. Los resultados se pueden observar a continuación en la Tabla 1. 


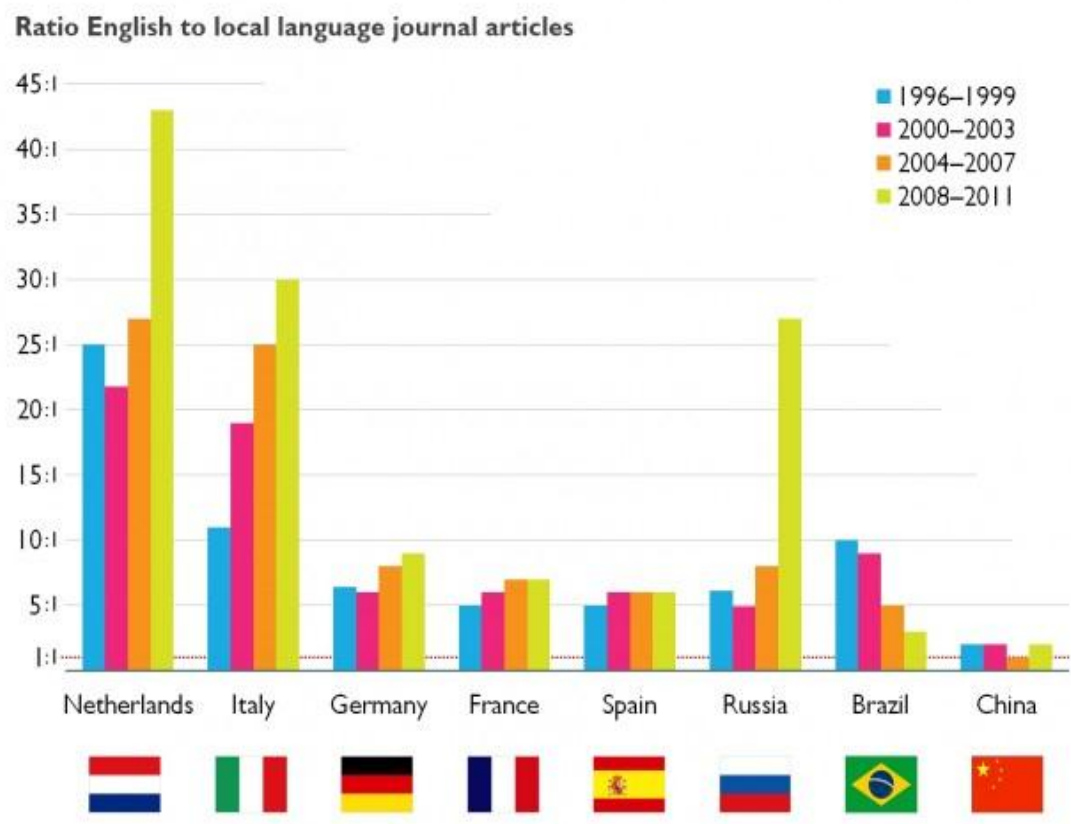

Figura 2. Número de artículos publicados por investigadores en inglés y en otros idiomas oficiales de ocho diferentes países en revistas especializadas entre 19962011. (Fuente: Scopus. Tomado de VAN WEIJEN, 2012)

Tabla 1. Participación del idioma inglés de acuerdo con diferentes campos de la ciencia. Fuente: VAN WEIJEN (2012)

\begin{tabular}{|c|c|c|c|c|c|}
\hline \multirow[b]{2}{*}{ Language } & \multicolumn{2}{|c|}{ "Hard" Sciences } & \multicolumn{2}{|c|}{ "Soft" Sciences } & \multirow{2}{*}{$\begin{array}{c}\text { Multi- } \\
\text { disciplinary } \\
\& \\
\text { Undefined }\end{array}$} \\
\hline & $\begin{array}{c}\text { Life } \\
\text { Sciences }\end{array}$ & $\begin{array}{l}\text { Physical } \\
\text { Sciences }\end{array}$ & $\begin{array}{l}\text { Health } \\
\text { Sciences }\end{array}$ & $\begin{array}{c}\text { Social } \\
\text { Sciences, Arts } \\
\text { \& Humanities }\end{array}$ & \\
\hline English & 23.4 & 44.7 & 19.5 & 10.7 & 1.7 \\
\hline Chinese & 8.7 & 72.5 & 13.0 & 2.9 & 2.9 \\
\hline Dutch & 14.9 & 3.2 & 52.3 & 26.1 & 3.5 \\
\hline French & 8.6 & 16.3 & 36.4 & 36.5 & 2.3 \\
\hline German & 7.3 & 34.5 & 32.5 & 23.5 & 2.2 \\
\hline Italian & 4.7 & 12.1 & 38.6 & 40.6 & 4.0 \\
\hline Portuguese & 26.1 & 11.5 & 38.4 & 22.1 & 1.9 \\
\hline Russian & 17.2 & 45.0 & 21.0 & 8.4 & 8.4 \\
\hline Spanish & 10.8 & 13.2 & 44.4 & 29.6 & 2.0 \\
\hline
\end{tabular}


Estos resultados indican que los investigadores que publican en inglés, chino y ruso tienden a publicar más en campos relacionados con las ciencias duras 0 puras como la física, ingenierías y ciencias materiales. Por otro lado, los investigadores quienes escogen publicar en holandés, francés, italiano, portugués y español tienden a publicar sus trabajos relacionados con campos de las ciencias humanas o blandas, como las de la salud, las sociales, psicología, artes y humanidades. Este oscila casi del $80 \%$ para Holanda e Italia y hasta $60 \%$ para Alemania y Portugal. Aunque estos rangos son similares en casi todos los países hay un alto nivel de variación en los campos actuales dentro de estas categorías. Por ejemplo, más de la mitad de toda la publicación del idioma holandés en relación con las ciencias de la salud, la cual incluye medicina, odontología, enfermería y veterinaria, mientras que en italiano casi $41 \%$ de todas las publicaciones están relacionadas con ciencias sociales, artes y humanidades VAN WEIJEN (2012).

Continuando con la revisión, se encontró otro interesante estudio adelantado por VAN LEEUWEN et al. (2001), en esta investigación se buscó respuesta a la pregunta: ¿De qué manera se comparan el impacto de los documentos escritos en idiomas diferentes al inglés en comparación con los escritos en dicha lengua?. Los resultados muestran que el $20 \%$ de todas las publicaciones de la facultad de medicina de la Universidad de Münster (Alemania) correspondía a publicaciones de idiomas diferentes al inglés y el $80 \%$ a investigaciones en inglés. Aquí encontramos otra vez el predominio del inglés incluso en la investigación de una sola universidad. Las Figs. 3,4 y 5 , se muestra que el impacto de las publicaciones escritas en otros idiomas diferentes al inglés es más bajo. Esto los llevó a la conclusión de que el impacto de las investigaciones de esa facultad de medicina se incrementa debido al promedio del nivel mundial ya que los documentos están escritos en inglés. Las siguientes figuras relacionan los resultados de las investigaciones de cinco países: Estados Unidos, Reino Unido, Francia, Alemania y Suiza en el periodo 1981-1998. Los resultados del análisis indican claramente la modesta posición de Alemania y Francia. Alemania alcanza un impacto ligeramente superior al nivel mundial solamente en el más reciente bloque de años (VAN WEIJEN; 2012).

En el mismo estudio, VAN LEEUWEN et al. (2001) presentan una crítica acerca del hecho que hay un gran número de publicaciones científicas de grandes países como Alemania y Francia que no son cubiertas por la Science Citation Index (SCl). Este fenómeno en su opinión podría subestimar los resultados científicos de estas naciones y explicar el bajo y modesto impacto de los mismos. 


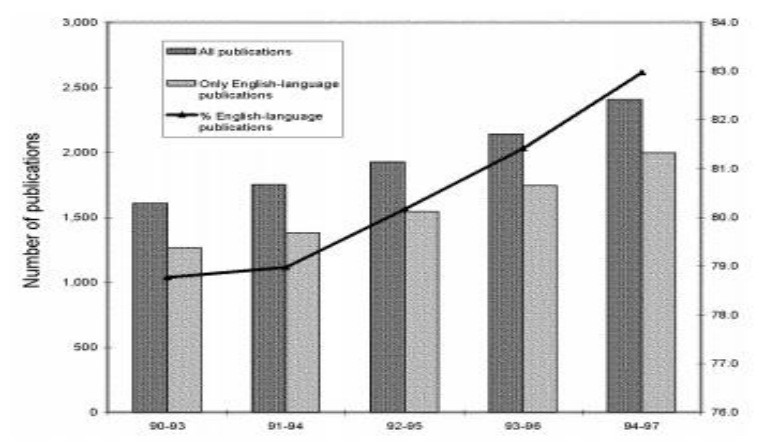

Figura 3. Comparación de los resultados de la Facultad de medicina de la Universidad de Münster (Alemania) de todas las publicaciones hechas en inglés y en otros idiomas. Fuente: VAN LEEUWEN et al. (2001)

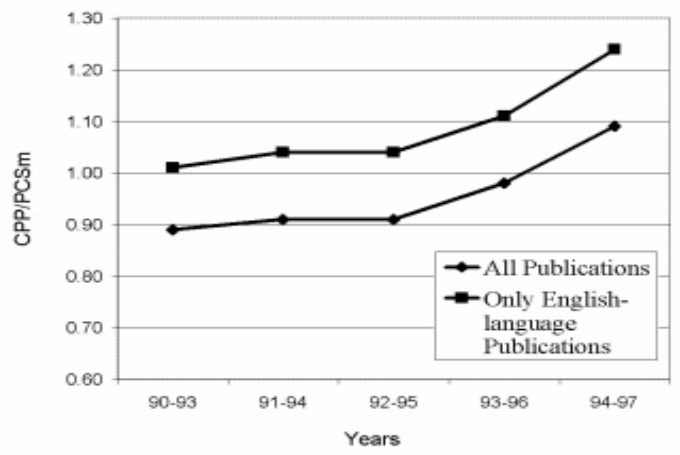

Figura 4. Impacto de los resultados de las publicaciones hechas por la Facultad de Medicina de la Universidad de Münster (Alemania) en todos los idiomas y solo en el idioma inglés entre 1990-1997. Fuente: VAN LEEUWEN et al. (2001)

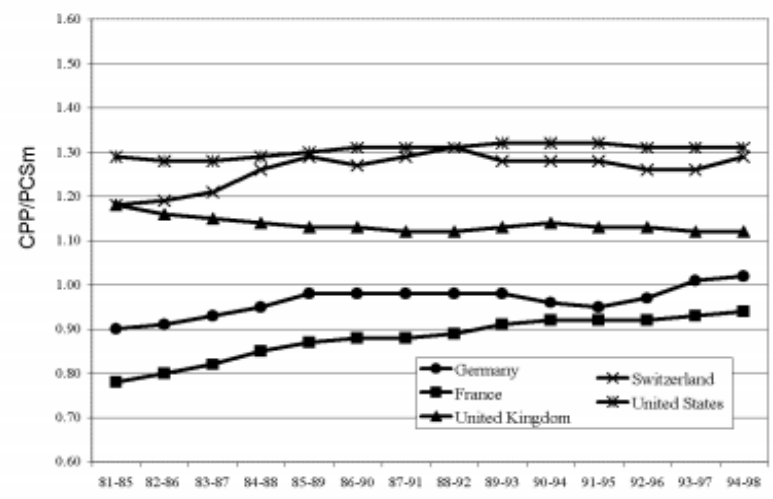

Figura 5. Análisis del impacto de los cinco sistemas médicos $R \& D$, de toda las publicaciones cubiertas por la revista $\mathrm{SCl}$ sobre investigación médica. Fuente:

VAN LEEUWEN et al. (2001) 
A estas alturas suenan muy adecuadas las palabras de Beatriz Cabanillas, una becaria postdoctoral, sobre el inglés en el ámbito científico: "...En este campo estoy dándome cada vez más cuenta de lo importante que es manejar una única lengua para poder contar al mundo lo que estás haciendo en tu laboratorio, lo que estás consiguiendo y sobre todo, conocer los hallazgos que se producen día a día en tu campo de investigación en otras partes del mundo. La vía fundamental para expandir estos conocimientos son sin duda las revistas científicas. $Y$ si bien es cierto que algunas revistas científicas en español tienen cada vez mayores índices de impacto, no cabe ninguna duda de que son las revistas en inglés el motor fundamental de la ciencia. Pienso que todos los que estamos vinculados a este campo somos profundamente conscientes de este hecho. Hecho que para un grandísimo porcentaje es un hecho molesto...molesto, porque cuesta esfuerzo. Cuesta tener que leer las revistas en inglés con los hallazgos previos en tu investigación para saber lo que ya está hecho y lo que aún está por hacer. Cuesta tener que escribir tus resultados en un buen inglés para que puedan ser aceptados en una revista científica. Cuesta tener que preparar pósters en inglés y sobre todo comunicaciones orales con tus resultados para presentarlos en los congresos anuales internacionales de tu campo de investigación...pero sobre todo, lo que creo que no cabe ninguna duda que más esfuerzo cuesta ( $y$ en este punto estaría de acuerdo conmigo la gran mayoría, incluso los más experimentados) es interaccionar con otros colegas en esos mismos congresos y más si eres tú el que está subido al atril y eres el foco al que se dirigen las preguntas en inglés con cualquier acento de cualquier país del mundo. Creo que ésta es la realidad. Todos somos conscientes de ello y es algo que nos punza y nos molesta cada poco tiempo. $Y$ por supuesto que yo no soy una excepción y soy a la primera que le cuesta, que le punza y que le molesta".

\section{Recomendaciones}

En el presente artículo se confirma que el inglés es el idioma preferido por los investigadores al momento de publicar sus hallazgos, la mayor parte de la investigación científica es inicialmente publicada en revistas especializadas en inglés, consideradas fuentes primarias de información; sin embargo, aunque el inglés parece ser el idioma favorito, algunas investigaciones también se publican en otros idiomas. Es recomendable publicar los resultados de investigaciones en revistas internacionales y en la medida de lo posible en una lengua que llegue a más lectores. Un idioma común facilita el intercambio de conocimientos y experiencias.

El creciente aumento de la investigación en diferentes áreas del saber nos lleva a la necesidad de actualización constante, en pro de los avances de la ciencia y la 
tecnología. Para sobrevivir en este mundo competitivo, todos debemos ser capaces de asumir nuevos retos y oportunidades. A través del uso de internet y los teléfonos celulares, el mundo se ha convertido en una pequeña aldea, donde el primero que recibe la información sobresaldrá ante aquellos que nunca la reciban o la reciban muy tarde. ¿Qué idioma nos permite una comunicación a gran escala? ... El inglés, por supuesto.

\section{Referencias}

CRYSTAL, D. 1977. The Cambridge Encyclopedia of the English Language. Cambridge University Press. Cambridge, UK.

CRYSTAL, D.; 2004. The Stories of English. Allen Lane. London, UK. Disponible en: $\quad$ http://www.privateacher.edu.pe/Boletin.asp?Articulold=0501 Historialngles. Consultado: 28-03-2013.

KATSIAVRIADES, K.; QURESHI, T. 2002. The Origin and History of the English Language. Disponible en: http://www.krysstal.com/english.html. Consultado: 29-032013.

KIRCHIK, O.; GINGRAS, Y.; LARIVIÈRE, V. 2012. Changes in publication languages and citation practices and their effect on the scientific impact of Russian science (1993-2010). Journal of the American Society for Information Science and Technology 63 (7):1411-1419.

KRYSS K.; TALAAT, Q. 2002. The Origin and History of the English Language. Disponible en: http://www.privateacher.edu.pe/Boletin.asp?Articulold=0501 Historialngles.

Consultado: 30-03-2013.

SUBEDI, D. 2010. English and Scientific Research: Some Reflections | Nelta Choutari). Disponible en: www.neltachoutarywordpress.com. Consultado: 01-042013.

TARDY, C. 2004. The role of English in scientific communication: lingua franca or tyrannosaurus rex?. Journal of English for academic purposes 3(3):247-269.

THOMPSON, W.F.; BALKWILL, L.L. 2006. Decoding speech prosody in five languages. Semiótica 2006 (158). 407-424.

VAN LEEUWEN, T. N.; MOED, H.F.; TIJSSEN, R.J.W.; VISSER, M.S.; VAN RAAN, A.F.J. 2001. Language biases in the coverage of the Science Citation Index and its consequences for international comparisons of national research performance. Scientometrics 51 (1): 335-346.

VAN WEIJEN, D. 2012. The Language of (Future) Scientific Communication. Research Trends 31. Disponible en: http://www.researchtrends.com/issue-31- 
november-2012/the-language-of-future-scientific-communication/. Consultado: 0203-2013.

WATERS, J. 2004. Whither English? The Washington Times. (Sept. 16, 2004). USA. 